\title{
Elevated Sympathetic Nerve Activity in Patients with Accelerated Essential Hypertension
}

\author{
Toshiyoshi Matsukawa, ${ }^{*}$ Tadaaki Mano, ${ }^{*}$ Eiji Gotoh, ${ }^{\star}$ and Masao Ishii ${ }^{\star}$ \\ * Department of Autonomic and Behavioral Neurosciences, Division of Higher Nervous Control, Research Institute of Environmental \\ Medicine, Nagoya University, Nagoya 464-01, Japan; and ${ }^{\ddagger}$ Second Department of Internal Medicine, Yokohama City University School \\ of Medicine, Yokohama 236, Japan
}

\begin{abstract}
To determine if an abnormality exists in the sympathetic nervous system of patients with accelerated hypertension, we recorded muscle sympathetic nerve activity (MSNA) from the tibial nerve by microneurography in eight benign essential hypertensives and seven accelerated essential hypertensives. Basal MSNA, plasma renin activity, and plasma angiotensin II levels were significantly higher in accelerated hypertensives than in benign hypertensives $(P<0.05)$. To clarify the relationship between the renin-angiotensin axis and sympathetic nervous system in the accelerated hypertensives, we measured the MSNA after $7 \mathrm{~d}$ of oral administration of captopril ( 75 $\mathrm{mg} / \mathrm{d}$ ) for antihypertensive treatment in the benign hypertensives and accelerated hypertensives. After administering captopril, the arterial pressure decreased significantly in the benign hypertensives and accelerated hypertensives with decreases in plasma angiotensin II levels, and the decreases in arterial pressure were greater in the accelerated hypertensives than in the benign hypertensives. After captopril administration, the MSNA decreased significantly in the accelerated hypertensives but did not change in the benign hypertensives. Thus, in accelerated hypertensives, sympathetic tone is elevated, and the elevated sympathetic tone is closely related to the activated renin-angiotensin axis tone. (J. Clin. Invest. 1993. 92:25-28.) Key words: accelerated hypertension • renin-angiotensin axis • captopril • essential hypertension - muscle sympathetic nerve activity
\end{abstract}

\section{Introduction}

Malignant hypertension remains one of the most serious complications of hypertensive disease. There are several theories regarding the mechanisms underlying the development of malignant hypertension. The activation of sympathetic nerve activity was reported recently, to accelerate hypertension and thereby induce malignant hypertension both in human $(1,2)$ and animal (3). The elevation of sympathetic nerve activity is suggested to play a role in the development of essential hypertension by studies in which muscle sympathetic nerve activity

Address correspondence to Toshiyoshi Matsukawa, M.D., Department of Autonomic and Behavioral Neurosciences, Division of Higher Nervous Control, Research Institute of Environmental Medicine, Nagoya University, Furo-cho, Chikusa-ku, Nagoya 464-01, Japan.

Received for publication 29 July 1992 and in revised form 8 February 1993.

J. Clin. Invest.

(c) The American Society for Clinical Investigation, Inc.

$0021-9738 / 93 / 07 / 0025 / 04 \$ 2.00$

Volume 92, July 1993, 25-28
(MSNA) ${ }^{1}$ was measured directly by microneurography $(4,5)$. Matsukawa et al. and Anderson et al. have reported recently that the MSNA at rest $(4,5)$ and in response to stressors (4) are greater in young borderline hypertensives than in normotensives. Also in benign essential hypertensives, MSNA at rest has been shown to be mildly elevated as compared with normotensives in our previous study (6). If, in essential hypertensives, the sympathetic nerve activity is more exaggerated, their arterial pressures can be more readily elevated. However, there has been so far no systematic study in which sympathetic nerve activity was recorded directly in subjects with accelerated or malignant hypertension.

The activation of the renin-angiotensin axis is known to play a very important role in the pathogenesis and pathophysiology of accelerated or malignant hypertension (7, 8). Angiotensin II (ANG II) is known to stimulate the sympathetic nervous system at the level of the adrenergic nerve ending $(9,10)$, the sympathetic ganglia (11) and the central nervous system (12-14). Our previous studies demonstrated that an intravenous infusion of ANG II stimulates MSNA in humans (15, 16). Thus, in essential hypertension, the stimulatory effect of angiotensin II on sympathetic nervous system may play a role in the development of malignant hypertension. The relationship, however, between the endogenous renin-angiotensin system and the sympathetic nervous system has not yet been defined precisely in accelerated or malignant hypertension.

In the present study, we first measured the resting basal MSNA in benign and accelerated essential hypertensives. Secondly, to elucidate the interactions between the endogenous renin-angiotensin and sympathetic nervous systems, we examined the effect of the angiotensin converting enzyme inhibitor, captopril, on the MSNA in these subjects.

\section{Methods}

Subjects. We studied eight benign essential hypertensives (four males and four females; $40 \pm 3$ years) and seven accelerated essential hypertensives (four males and three females; $39 \pm 2 \mathrm{yr}$ ) who participated in a single-blind trial of placebo and captopril. After admission to our hospital, all patients were first administered a placebo orally for 3-7 d, and on the last day, the microneurographic study was made. Then captopril was administered orally for $7 \mathrm{~d}(75 \mathrm{mg} / \mathrm{d})$, and the same study was performed on the last day. All hypertensives were placed on a diet containing $110 \mathrm{meq} / \mathrm{d}$ of sodium chloride. Patients were considered to have benign essential hypertension if their sitting diastolic blood pressure was $>95 \mathrm{mmHg}$ and $<105 \mathrm{mmHg}$ at the outpatient clinic on three different occasions. In these subjects, plasma renin activity levels after resting supine for $30 \mathrm{~min}$ ranged from 0.5 to $2.4 \mathrm{ng} / \mathrm{ml} \mathrm{per} \mathrm{h}$. All benign hypertensives had never been treated and had not received any

1. Abbreviations used in this paper: ANG II, angtiotensin II; MSNA, muscle sympathetic nerve activity; PRA, plasma renin activity. 
medications before the study. After admission to our hospital, they were given a placebo for $7 \mathrm{~d}$ before the study.

The diagnosis of accelerated hypertension was based on a markedly elevated diastolic arterial pressure, $>130 \mathrm{mmHg}$, and retinal hemorrhages and exudates or papilledema. Two patients had grade IV retinopathy (Keith-Wagener criteria), and three patients had grade III retinopathy. There were two patients with grade Ilb retinopathy. Patients who had accelerated hypertension with renal insufficiency (serum creatinine levels $>1.6 \mathrm{mg} / \mathrm{dl}$ ) were not included in this study since it was difficult to exclude the possibility that these patients had a component of renal hypertension. All patients had been administered calcium channel blockers, nifedipine or diltiazem, before admission at the outpatient clinic. After admission to our hospital, the antihypertensive drugs were discontinued and placebo was administered for 3-7 d: two patients with grade IV retinopathy and two patients with grade III retinopathy received placebo for $3 \mathrm{~d}$, one patient who had grade III retinopathy and a diastolic blood pressure of $<120 \mathrm{mmHg}$ received placebo for $5 \mathrm{~d}$, and two patients with grade IIb retinopathy received placebo for $7 \mathrm{~d}$. During the administration of placebo, endocrinological examinations were made to exclude secondary hypertension, and then the present study was performed on the last day. Since we preliminarily found no significant differences between before therapy and $3 \mathrm{~d}$ after stopping therapy with calcium channel blockers for $2 \mathrm{wk}$ in either blood pressure (nifedipine, $173 \pm 5 / 108 \pm 4$ before vs $168 \pm 5 / 105 \pm 4 \mathrm{mmHg}$ after, $n$ = 5; diltiazem, $161 \pm 4 / 102 \pm 3$ before vs $165 \pm 4 / 104 \pm 3 \mathrm{mmHg}$ after, $n$ $=5$ ) or MSNA (nifedipine, $36 \pm 5$ before vs. $34 \pm 4$ bursts/min after, $n$ $=5$; diltiazem, $32 \pm 4$ before vs. $34 \pm 4$ bursts $/ \min$ after, $n=5$ ), we made the recording of MSNA $\geq 3 \mathrm{~d}$ after stopping the therapy.

Secondary hypertension was ruled out through screening examinations on all patients with benign and accelerated hypertension. The examinations undertaken on these patients included blood counts, urinalysis, urine culture, serum and urine electrolytes, plasma renin activity, $24 \mathrm{~h}$ urine for metanephrines, cortisol and aldosterone, and rapidsequence intravenous urography or dynamic renal flow scan with technetium-99m diethylenetriaminepentaacetic acid.

Written informed consent was obtained from each subject following a detailed explanation of the purpose of the study, the procedures and possible complications. The protocols had been approved by the Human Studies Committee of our department.

Procedures. Subjects were examined while supine throughout the study. An antecubital vein was cannulated for blood sampling. Arterial pressure was measured using an automatic sphygmomanometer (1846SX; Critikon, Tampa, FL). Heart rate and respiration were continuously monitored. The MSNA was recorded continuously using the microneurographic method $(4,5,16,17)$.

Recording of MSNA. Multiunit recordings of the MSNA were obtained from a muscle fascicle of the tibial nerve at the popliteal fossa. To record the MSNA, a tungsten microelectrode, $100 \mu \mathrm{m}$ in diameter, with an uninsulated tapered tip of 1-5 $\mu \mathrm{m}$ and an impedance of $\sim 5$ M $\Omega$ (model no. 26-05-1; Federick Haer \& Co., Brunswick, ME) was inserted manually through the skin. Spike potentials were amplified (DPA-201 and DPA-200; DIA Medical, Tokyo) and monitored visually on an oscilloscope (VC-10; Nihon Kohden, Tokyo) and audibly on a speaker. The amplified neurogram was fed through a bandpass filter (FV-664; NF Electronic Instruments, Tokyo) with a band width of $400-3,000 \mathrm{~Hz}$. Next, the filtered neurogram was passed through an integrator (model no. 1333; NEC-San-ei, Tokyo) with a time constant of $0.1 \mathrm{~s}$ to obtain the mean voltage neurogram of $\operatorname{MSNA}(4,5,16,17)$.

The spikes were identified as corresponding to the MSNA according to the criteria defined in previous studies $(4,17)$ by the following criteria: $(a)$ tapping or stretching the muscle and tendon supplied by the impaled fascicle of the tibial nerve elicited afferent mechanoreceptor discharge, whereas stroking the skin innervated by the tibial nerve did not; and $(b)$ spikes revealed a characteristic pulse-synchronous "spontaneous" discharge during phases II and III of Valsalva's maneuver

Quantitative analysis of MSNA. For quantitative analysis, the mean voltage neurogram of MSNA was displayed together with the electrocardiogram on a multidot thermal recorder ( 8 M14; San-ei, Tokyo). Records were divided into periods of $1 \mathrm{~min}$ each, and the number of sympathetic bursts was determined from the mean voltage neurogram and expressed as burst frequency (bursts/min) $(4,17)$. Because the burst frequency was considered to be the best index of MSNA for interindividual comparative studies, this parameter was used to compare basal levels of MSNA between groups.

Protocol. After the subject had rested supine for $30 \mathrm{~min}$ and after the MSNA was recorded successfully, the resting basal values of arterial pressure, heart rate and MSNA were measured and recorded for 15 min. To determine the basal levels of plasma renin activity (PRA) and plasma ANG II, blood samples were obtained via the venous cannula. After $7 \mathrm{~d}$ of oral administration with captopril $(75 \mathrm{mg} / \mathrm{d})$ arterial pressure, heart rate, MSNA, and plasma ANG II levels were measured again in eight benign hypertensives and five of seven accelerated hypertensives.

Measurement of PRA and the plasma concentration of ANG II. Blood samples were collected in chilled EDTA-2Na tubes and promptly centrifuged. PRA and the plasma ANG II concentration were measured using a radioimmunoassay.

Statistics. Data were expressed as mean \pm SEM. The statistical differences between groups for each parameter were assessed by paired and unpaired Student's $t$ tests. Statistical significance was defined as $P$ $<0.05$.

\section{Results}

Basal values of arterial pressure, heart rate, PRA, plasma ANG II concentration, and MSNA. Arterial pressures taken at rest during the study were significantly higher in the accelerated hypertensives than in the benign hypertensives (Table I). The PRA and plasma ANG II were significantly higher in the accelerated hypertensives than in the benign hypertensives. The heart rate in the accelerated hypertensives did not differ from that in benign hypertensives (Table I). The basal MSNA was significantly higher in the accelerated hypertensives than in the benign hypertensives $(P<0.05$, Fig. 1$)$.

Effect of the angiotensin converting enzyme inhibitor, captopril, on arterial pressure, heart rate, and MSNA. $7 \mathrm{~d}$ after the oral administration of captopril $(75 \mathrm{mg} / \mathrm{d})$, the plasma ANG II decreased significantly in the benign hypertensives and accelerated essential hypertensives (Table II). Captopril significantly decreased the arterial pressure in both benign and accelerated hypertensives. The depressor response was significantly greater in accelerated hypertensives than in benign hyperten-

Table I. Basal Systolic and Diastolic Arterial Pressures, Heart Rate, PRA, and Plasma ANG II in Benign and Accelerated Essential Hypertensives

\begin{tabular}{lcc}
\hline \multicolumn{1}{c}{ Variables } & $\begin{array}{c}\text { Benign } \\
\text { hypertensives } \\
(n=8)\end{array}$ & $\begin{array}{c}\text { Accelerated } \\
\text { hypertensives } \\
(n=7)\end{array}$ \\
\hline Age (yr old) & $40 \pm 3$ & $39 \pm 2$ \\
Arterial pressure $(\mathrm{mmHg})$ & $161 \pm 4$ & $210 \pm 3^{*}$ \\
$\quad$ Systolic & $91 \pm 2$ & $126 \pm 3^{*}$ \\
$\quad$ Diastolic & $60 \pm 2$ & $68 \pm 5$ \\
Heart rate (beats $\left.\cdot \mathrm{min}^{-1}\right)$ & $1.4 \pm 0.3$ & $6.5 \pm 1.0^{*}$ \\
PRA (ng ANG I $\left.\cdot \mathrm{ml}^{-1} \cdot \mathrm{h}^{-1}\right)$ & $19 \pm 4$ & $59 \pm 5^{*}$ \\
Plasma ANG II $\left(\mathrm{pg} \cdot \mathrm{ml}^{-1}\right)$ & & \\
\end{tabular}

Data are expressed as mean \pm SEM. ${ }^{*} P<0.05$ vs benign hypertensives. 


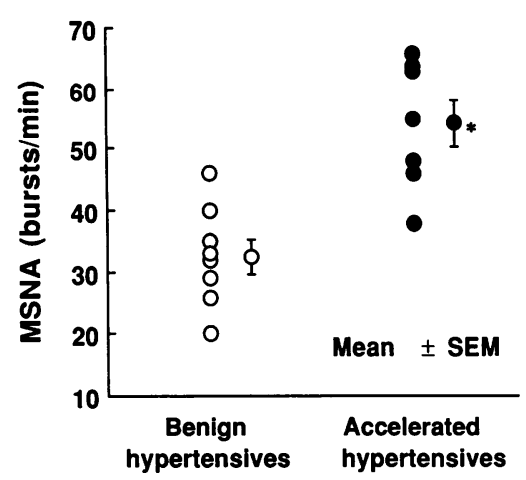

sives. The heart rate did not change significantly in any of the two groups (Table II). The MSNA decreased significantly in accelerated hypertensives, but did not change significantly in benign hypertensives (Fig. 2).

\section{Discussion}

In this study, we measured the sympathetic nerve activity directly in benign and accelerated essential hypertensives. Two key observations were made. First, not only the renin-angiotensin axis tone, but the basal sympathetic neural tone was also markedly elevated in accelerated hypertensives as compared with benign hypertensives. Secondly, in the accelerated hypertensives, the sympathetic nerve activity was decreased during administration of captopril associated with decreases in the renin-angiotensin axis tone.

An abnormality of the sympathetic nervous system is believed to be involved in the pathogenesis of essential hyperten-

Table II. Systolic and Diastolic Arterial Pressures, Heart Rate, and Plasma ANG II in Benign and Accelerated Essential Hypertensives during Administration of Placebo and during Administration of Captopril

\begin{tabular}{lcc}
\hline \multicolumn{1}{c}{ Variables } & $\begin{array}{c}\text { Benign } \\
\text { hypertensives } \\
(n=8)\end{array}$ & $\begin{array}{c}\text { Accelerated } \\
\text { hypertensives } \\
(n=5)\end{array}$ \\
\hline $\begin{array}{l}\text { Age (yr old) } \\
\text { Arterial pressure }(\mathrm{mmHg})\end{array}$ & $40 \pm 3$ & $38 \pm 3$ \\
$\quad \begin{array}{l}\text { Systolic } \\
\quad \text { Placebo }\end{array}$ & $161 \pm 4$ & \\
$\quad$ Captopril & $146 \pm 4^{\ddagger}$ & $185 \pm 3^{*}$ \\
$\quad$ Diastolic & & 185 \\
$\quad$ Placebo & $91 \pm 2$ & $126 \pm 3^{*}$ \\
$\quad$ Captopril & $83 \pm 2^{\ddagger}$ & $105 \pm 3^{* \ddagger}$ \\
Heart rate (beats $\left.\cdot \mathrm{min}^{-1}\right)$ & $64 \pm 2$ & $74 \pm 4$ \\
$\quad$ Placebo & $66 \pm 2$ & $70 \pm 5$ \\
$\quad$ Captopril & & $63 \pm 5^{*}$ \\
Plasma ANG II $\left(\mathrm{pg} \cdot \mathrm{ml}^{-1}\right)$ & $19 \pm 4$ & $19 \pm 4^{\ddagger}$ \\
Placebo & $9 \pm 5^{\ddagger}$ & \\
Captopril & & \\
\hline
\end{tabular}

Data are expressed as mean \pm SEM.

${ }^{*} P<0.05$ vs. benign hypertensives.

${ }^{\ddagger} P<0.05$ vs. placebo.

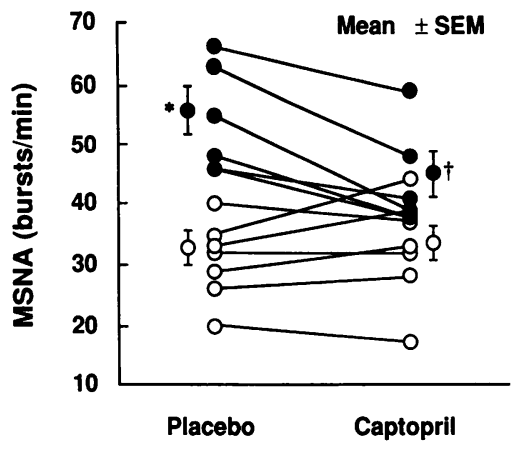

Figure 2. Individual and mean data showing MSNA at rest in the benign essential hypertensives $(0)$ and accelerated essential hypertensives $(\bullet)$ during administration of placebo and during administration of captopril. ${ }^{*} P<0.05$ vs benign essential hypertensives. ${ }^{\dagger} P<0.05$ vs placebo.

The basal MSNA was greater in the accelerated hypertensives than in the benign hypertensives. The MSNA decreased significantly in the accelerated hypertensives but did not change in the benign hypertensives after the administration of captopril.

sion. This evidence is derived mainly from studies of plasma norepinephrine. It does not, however, allow one to determine whether the elevated plasma norepinephrine results from facilitated release or impaired uptake of norepinephrine at the adrenergic nerve ending, or whether it results from an increase in central sympathetic neural outflow. Direct measurements of MSNA by microneurography (17) have provided some insights into the question of whether sympathetic neural outflow is augmented in hypertension. It has been demonstrated that MSNA is elevated in young borderline hypertensives $(4,5)$ and in mildly essential hypertensives (6), suggesting that the elevation of sympathetic nerve activity contributes to the development and maintenance of essential hypertension. The more activation of sympathetic nerve activity has been suggested to be related to the pathogenesis in accelerated or malignant hypertension in both human $(1,2)$ and animal (3) studies. Until now, there has been no study in which the sympathetic nerve activity in accelerated or malignant hypertensives was measured directly. By direct measurement, in this study, we demonstrated that the sympathetic neural outflow is markedly elevated in accelerated hypertensives, and we confirmed the hypothesis that the sympathetic nervous system plays a role in the development of malignant hypertension.

In accelerated hypertensives, we also found that the elevated sympathetic neural outflow is reduced by an administration of captopril with a decrease in plasma levels of ANG II. This also agrees with previous studies $(18,19)$ that have shown that captopril decreases significantly the plasma norepinephrine in hypertensive patients $(18,19)$, and that the magnitude of the decrease in norepinephrine levels induced by captopril correlates with the pretreatment level of norepinephrine (19). That is, the levels of plasma norepinephrine, which may reflect sympathetic nerve activity, in the hypertensives could depend on the endogenous renin-angiotensin axis tone. The activation of renin-angiotensin axis is known to play a very important role in the pathogenesis of accelerated or malignant hypertension $(7,8)$. ANG II has been shown to stimulate the sympathetic nervous system both at the level of the sympathetic ganglia (11) and central nervous system (12-14) in animals as well as in humans. We also found in our previous study that ANG II accelerated the MSNA (16). Thus, we suggest that the activated renin-angiotensin axis tone in hypertensives participates in the elevation of sympathetic neural outflow in accelerated hypertensives. 
In conclusion, sympathetic neural outflow to muscle was elevated in accelerated hypertension. Thus, the sympathetic nervous system seems to play an important role in the development of malignant hypertension. Furthermore, the enhanced endogenous renin-angiotensin axis tone may mediate the increase in sympathetic neural outflow seen in accelerated hypertensives.

\section{Acknowledgments}

This work was supported in part by Grants for Scientific Research from the Ministry of Education, Science and Culture of Japan (60440048).

\section{References}

1. Sesoko, S., N. Miyazaki, Y. Kato, O. Tochikubo, and Y. Kaneko. 1984. Atropine and a hypertensive crisis. Ann. Intern. Med. 101:720.

2. Sesoko, S., N. Akema, T. Matsukawa, and Y. Kaneko. 1987. Predisposing factors for the development of malignant essential hypertension. Arch. Intern. Med. 147:1721-1724.

3. Sesoko, S., H. Iwasawa, N. Akema, T. Matsukawa, K. Sugiyama, H. Shionoiri, and Y. Kaneko. 1988. Sequential changes in urinary catecholamine in the transition from benign to malignant hypertension in rats. Jpn. Circ. J. 52:197201 .

4. Matsukawa, T., E. Gotoh, S. Uneda, E. Miyajima, H. Shionoiri, O. Tochikubo, and M. Ishii. 1991. Augmented sympathetic nerve activity in response to stressors in young borderline hypertensive men. Acta Physiol. Scand. 141:157165.

5. Anderson, E. A., A. S. Christine, W. J. Lawton, and A. L. Mark. 1989. Elevated sympathetic nerve activity in borderline hypertensive humans. Hypertension (Dallas). 14:177-183.

6. Yamada, Y., E. Miyajima, O. Tochikubo, T. Matsukawa, and M. Ishii. 1989. Age-related changes in muscle sympathetic nerve activity in essential hypertension. Hypertension (Dallas). 13:870-877.
7. Laragh, J. H., S. Ulick, W. Januszewicz, Q. B. Deming, W. G. Kelly, and S. Lieberman. 1960. Aldosterone secretion and primary and malignant hypertension. J. Clin. Invest. 39:1091-1106.

8. Rosset, E., J. R. Scherrer, and R. Veyrat. 1973. Increased plasma renin substrate concentration in human malignant hypertension. Helv. Med. Acta. 37:235-250.

9. Zimmerman, G. B. 1981. Adrenergic facilitation by angiotensin: does it serve a physiological function? Clin. Sci. (Lond.). 60:343-348.

10. Hughes, J., and R. H. Roth. 1971. Evidence that angiotensin enhances transmitter release during sympathetic nerve stimulation. Br. J. Pharmacol. 41:239-255.

11. Lewis, R. D., and E. Reit. 1965. The action of angiotensin II and bradykinin on the superior cervical ganglion of the cat. J. Physiol. 179:538-553.

12. Bickerton, R. K., and J. P. Buckley. 1961. Evidence for a central mechanism in angiotensin induced hypertension. Proc. Soc. Exp. Biol. Med. 106:834836.

13. Severs, W. B., A. E. Daniels, and J. P. Buckley. 1967. On the central hypertensive effect of angiotensin II. Int. J. Neuropharmacol. 6:199-205.

14. Ferrario, C. M., P. L. Gildenberg, and J. W. McCubbin. 1972. Cardiovascular effects of angiotensin mediated by the central nervous system. Circ. Res. 555:257-62.

15. Matsukawa, T., E. Gotoh, E. Miyajima, Y. Yamada, H. Shionoiri, O. Tochikubo, and M. Ishii. 1988. Angiotensin II inhibits baroreflex control of muscle sympathetic nerve activity and the heart rate in patients with essential hypertension. J. Hypertens. 6(Suppl. 4):S501-S504.

16. Matsukawa, T., E. Gotoh, K. Minamisawa, M. Kihara, S. Ueda, H. Shionoiri, and M. Ishii. 1991. Effects of intravenous infusions of angiotensin II on muscle sympathetic nerve activity in humans. Am. J. Physiol. 261:R690-R696.

17. Wallin, B. G. 1983. Intraneural recording and autonomic function in man. In Autonomic Failure. R. Bannister, editor. Oxford University Press, London. pp. 36-51.

18. Mitchell, H. C., W. A. Pettinger, L. Gianotti, G. Reed, L. Kirk, L. Kuhnert, C. Matthews, and R. Anderson. 1983. Further studies of the hyperadrenergic state of treated hypertensives. effect of captopril. Clin. Exp. Hypertens. A5:1611-1627.

19. Weinberger, M. H. 1982. Role of sympathetic nervous system activity in the blood pressure response to long-term captopril therapy in severely hypertensive patients. Am. J. Cardiol. 49:1542-3. 